\title{
Pt nanocrystal Evolution in the Presence of Au(III)-Salts at Room Temperature: Formation of AuPt Heterodimers
}

\begin{abstract}
Room temperature syntheses of AuPt heterodimers are reported using a simple protocol. The role of oleylamine and Pt NCs in the reduction and nucleation of Au has been investigated. There are two unique aspects in this synthesis. First, the synthesis was conducted at room temperature, which enabled the heterodimer growth to progress at a slower rate and thus allowed monitoring of the Au nucleation process. Secondly, the presence of Pt NC seeds markedly accelerated the epitaxial growth of Au serving both as nucleation platform and as initial catalytic reducer of the $\mathrm{Au}^{+3}$ precursor. The growth of $\mathrm{Au}$ on $\mathrm{Pt}$ NCs was monitored at different times by UV-vis, HRTEM and XRD.
\end{abstract}

\section{Introduction}

In addition to the extensive study of single-composite metal and metal oxide nanocrystals (NCs), ${ }^{1-2}$ engineered hybrid NCs represent a novel class of structures where multiple inorganic materials are joined together in the absence of a mediator (i.e. a linking molecule) and bring about materials with novel properties. $^{3-5}$ While the most commonly studied geometries are core-shell NCs, special interest is also being directed towards asymmetrical heterodimers. ${ }^{6-17}$ Unlike core-shells, where the outer material is uniformly grown around the metallic core, heterodimers are grown anisotropically with two materials (such as metal/metal, ${ }^{10,13}$ semiconductor/metal, ${ }^{7 b, 15}$ metal oxide/metal ${ }^{6}$ and metal oxide/semiconductor ${ }^{14}$ ) epitaxially joined through a small interfacial area. This can occur at a specific facet or domain, which leads to different morphologies such as rods with a metal tip, ${ }^{11,17}$ dumbbells $^{9}$ and peanut-like ${ }^{6 \mathrm{~d}}$ structures. In those cases when the second metal reduces and nucleates on top of the pre-existing seeds, a series of factors such as slight lattice mismatch, large interfacial energy, immiscibility of the materials and the limited source of electron donation prevent multiple nucleation sites and uniform coating of the seeds. ${ }^{6-9}$ 
A common theme for most of the reported heterodimers to date is the use of a high temperature synthetic process in the presence of a reducing agent. For example, Manna et al. illustrated that size tunability of both metal domains is affected not only by the temperature of the reaction, but also by the size of the initial seeds, and the concentration ratio of the metals. The heterodimers were synthesized in the presence of dodecylamine as a reducing agent. ${ }^{8 b}$ In a more recent paper, Sun et al. showed how different sized Pt seeds and the solvent polarity can lead to morphological control of the AuPt heterostructure (i.e. pear-, peanut- and clover-like). ${ }^{6 \mathrm{~d}}$ This synthesis was also performed at high temperature, and in the presence of oleylamine as the reducer. In the present work, we show that the Pt seeds play a very important role for the nucleation and growth of Au on Pt NCs to form heterodimer structures at room temperature in the presence of oleylamine as the reducer. The Pt NCs drastically accelerates the reaction by acting both as seed platforms for the Au nucleation and possibly as a catalytic reducer when the initial concentration of $\mathrm{Au}^{+3}$ is high.

\section{Experimental Procedures}

Chemicals. Platinum (II) acetylacetonate [Pt(acac) 2 , 98\%] was purchased from Strem Chemicals and used as received. Gold(III) chloride trihydrate $\left(\mathrm{HAuCl}_{4} \cdot 3 \mathrm{H}_{2} \mathrm{O}, 99 \%\right)$, oleylamine (OAM, 70\%), 1,2dichlorobenzene anhydrous (DCB, 99\%), 1,2-hexadecanediol (HDD, 90\%), cobalt carbonyl moistened with 5-10\% hexane $\left[\mathrm{Co}_{2}(\mathrm{CO})_{8}, 90-95 \%\right]$ and 1-undecanol (UD, 98\%) were purchased from SigmaAldrich and used as received. All chemicals were stored and prepared for synthesis in a UNIlab MBraun glovebox unless otherwise stated.

Instrumentation. Transmission electron microscopy (TEM) analysis was performed on a JEOL 1010 with an accelerating voltage of $80 \mathrm{kV}$. High resolution TEM (HRTEM) analysis was performed on a JEOL 2100 with an accelerating voltage of $200 \mathrm{kV}$. X-ray diffraction (XRD) data were collected on a PANalytical X’Pert diffractometer using a $\mathrm{Cu} \mathrm{K} \alpha$ radiation source $(\lambda=1.541 \AA)$. In a typical experiment, the $2 \theta$ diffraction (Bragg) angles were measured by scanning the goniometer from $25^{\circ}$ to $100^{\circ}$ at a speed 
of $0.021^{\circ} \mathrm{s}^{-1}$. The samples were prepared by precipitating the NCs in the presence of methanol, and dried under nitrogen before smearing onto (510) silicon wafers ( $3^{\circ}$ off axis) for XRD analysis. The peak positions were determined using the X'Pert HighScore program and compared before and after baseline correction. UV-vis spectra were obtained with a Shimadzu UV-2401PC spectrophotometer, over the range of $200-800 \mathrm{~nm}$ with a $1 \mathrm{~cm}$ path length quartz cuvette. Baseline correction was performed and the spectra were stacked in order to compare the changes in the absorption peak. Pt analyses were performed using an Inductively Coupled Plasma Mass Spectrometry (ICP-MS) Agilent instrument (Model: 7500cx) with a detection limit of $0.02386 \mathrm{ppb}$. Aliquots of the samples were diluted to an optimal concentration for ICP-MS analysis. Ga was used as the internal standard and the integration time/point and time/mass were $0.1 \mathrm{~s}$ and $0.3 \mathrm{~s}$, respectively with a $3 \mathrm{x}$ repetition.

Synthesis of Pt NCs. The Pt NCs used in the AuPt heterodimer synthesis were synthesized and purified using a procedure described previously. ${ }^{18}$ Briefly, a solution containing 95 mg of HDD (0.37 mmol), 45 $\mu \mathrm{L}$ of OAM and $10 \mathrm{~mL}$ of DCB was added to a 3-neck round-bottom flask equipped with a condenser and stirred under an argon atmosphere. The solution temperature was increased to $180{ }^{\circ} \mathrm{C}$ using a glycerol oil bath over a time period of $\sim 30-60$ min. Once the boiling temperature of DCB was reached, a $3 \mathrm{~mL}$ solution of DCB containing $45 \mathrm{mg}$ of Pt(acac) $)_{2}(0.11 \mathrm{mmol})$ was added drop-wise at a rate of $0.5 \mathrm{~mL} / \mathrm{min}$. $100 \mu \mathrm{L}$ of Co trace prepared from $\mathrm{Co}_{2}(\mathrm{CO})_{8}(2 \mu \mathrm{mol})$ was then added immediately to the solution. The solution was refluxed at $180{ }^{\circ} \mathrm{C}$ for $20 \mathrm{~min}$ and gradually cooled down to room temperature before being transferred to an argon filled vial. The atomic Pt concentration immediately after synthesis and purification was $0.56 \mathrm{mg} / \mathrm{mL}$, as measured by ICP-MS. The concentration of Pt NCs was $1.21 \cdot 10^{14}$ NC/mL (calculated assuming cubic NCs of side length $6 \mathrm{~nm}$, Pt density of $21.45 \mathrm{~g} / \mathrm{cm}^{3}$ and atomic Pt concentration $0.56 \mathrm{mg} / \mathrm{mL})$.

Synthesis of AuPt heterodimers. A solution of previously purified Pt NCs in DCB (0.3 mL) was added to a solution of $1 \%$ of OAM in DCB $(2.7 \mathrm{~mL})$. A solution of $\mathrm{AuCl}_{4}{ }^{-}$in DCB (3 mL) $(0.79 \mathrm{mM}$ stock prepared with $5 \%$ UD to ensure complete dissolution of the $\mathrm{AuCl}_{4}^{-}$) was then added, and the resulting 
solution was stirred at room temperature for different time intervals. The final fractions of OAM and UD in the solution were $0.45 \%$ and $2.5 \%$ respectively. The growth of the heterodimers was monitored using TEM and UV-Vis and the solution was purged with $\mathrm{N}_{2}$ after each measurement, and stored in a darkened environment.

\section{Results and Discussion}

Effect of the reaction time. The growth of the Au domains on the Pt seeds was followed with time (Figure 1A). The solution initial appeared brownish due to the presence of Pt NC seeds, but it eventually evolved to pink/reddish after $24 \mathrm{~h}$. TEM samples taken at different reaction times show that the size of the growing Au NCs increased from $~ 8 \mathrm{~nm}(24 \mathrm{~h})$ to $\sim 9-10 \mathrm{~nm}(72 \mathrm{~h})$, and remained constant afterwards (Figure 1B). After $96 \mathrm{~h}$ of reaction, two types of heterodimer structures were observed, some having a peanut-like structure while the others showed a smooth merging between Au and Pt NCs. In addition, the initial Pt nanocubes develop into more spherical shapes with polished edges after forming the heterodimers (Figure S1, ESI), which is indicative of a higher reactivity of the vertex atoms in the Pt NCs and justifies the use of nanocubes for this reaction. It could also be appreciated a slight decrease of the initial Pt NC size (aprox. $1 \mathrm{~nm}$ ), when comparing with the measured diameter of the Pt domain in the heterodimers after more than 24 h (Figure 1C). However, we could not observe any further decrease of the size of these Pt domains, even after re-examining the samples a few months later. In order to evaluate the effect of an excess of $\mathrm{Au}^{+3}$ on the heterostructures, more $\mathrm{AuCl}_{4}^{-}$precursor was added to the reaction mixture after $196 \mathrm{~h}$ and stirred for an additional 4 days. Rather than obtaining multiple heterojunctions in each particle (i.e. peanut- or clover-like morphologies), we observed an increase in the size of the preexisting Au domains to $15.6 \mathrm{~nm}$, along with some free Au NCs.

Despite the bulk miscibility of Au and Pt, the heterodimer structure is thought to arise as a result of the electronic charge distribution within the seeds. ${ }^{6}$ As Au begins to nucleate on a particular facet of a Pt seed (most likely on the 111 surface), electrons are transferred from the Pt to the Au between their Fermi levels across the interface. ${ }^{6 b}$ The charge distribution is thus modified to compensate for the potential at the Au/Pt 
interface and the exposed Pt facets become much less effective points for Au nucleation. Also, when more $\mathrm{Au}^{+3}$ precursor is added to the existing heterodimers, the slight lattice mismatch tends to favor the growth of Au ions on Au NCs rather than on another facet of the Pt NCs. At room temperature, the required energy for Au nucleation is significantly higher than that for growth, further favoring the heterodimer formation. ${ }^{19}$ Hence, in our study, the Au will tend to nucleate on one of the Pt facets only. Please note that when $\mathrm{Au}$ NCs have been previously used as seeds, $\mathrm{Au}_{\text {core }}-\mathrm{Pt}_{\text {shell }}$ type structures resulted instead of heterodimers. $^{20}$

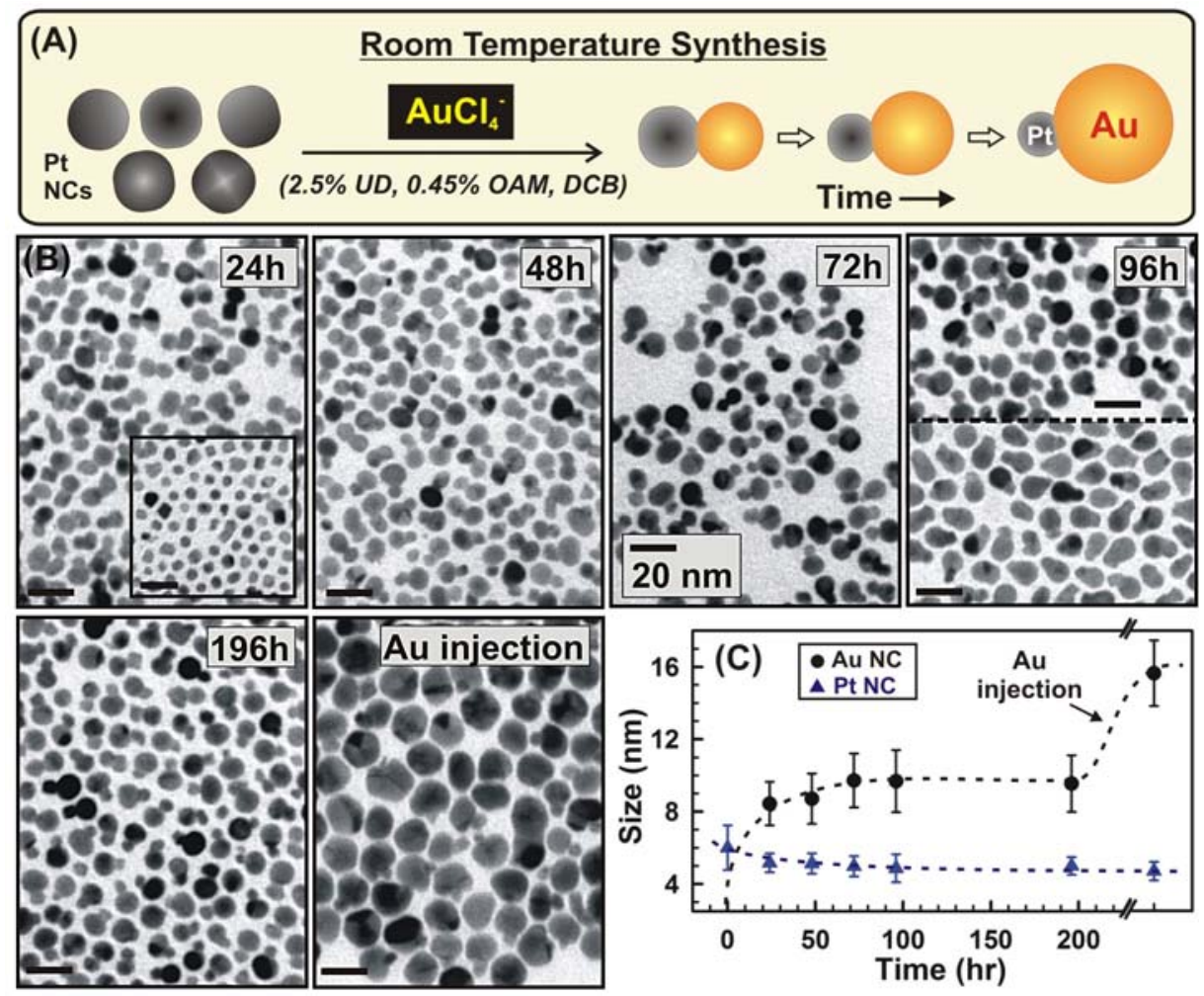

Fig. 1. (A) Schematic representation of the heterodimer formation, (B) TEM images and (C) plot of diameter vs. time for the heterodimer evolution. (Inset) TEM of the Pt NCs. All scale bars correspond to $20 \mathrm{~nm}$. The dotted lines illustrate the change in NC size and are not obtained from fitting. 
Role of Pt NCs. Control studies were performed to further substantiate the role of Pt seed in the heterodimer formation. When the experiment of figure 1 was repeated in the absence of Pt NCs, faint pinkish colored solutions could be observed only after 196 h (Figure S2, ESI). Analysis by TEM showed polydispersed Au NCs with non-spherical forms, thus confirming the role of the alkylamine as a reducer of the $\mathrm{Au}^{+3}$ precursor as it has already been reported previously. ${ }^{6 \mathrm{~d},}{ }^{8 b}$ However, the dramatic decrease of the reaction rate observed in this case (196 h) when comparing with the presence of Pt NCs (24 h), indicates that the Pt seeds strongly favor the nucleation of Au. This could be understood in terms of Pt NCs both i) acting as nucleation center and/or ii) catalyzing the reduction of $\mathrm{Au}^{+3}$ when the initial concentrations of $\mathrm{Au}^{+3}$ are very high. In principle, due to the noble nature of metallic $\mathrm{Au}\left(\mathrm{Au}^{+3} / \mathrm{Au}^{0}, E^{0}=\right.$ $1.51 \mathrm{~V})$, one would expect Pt NCs $\left(\mathrm{Pt}^{+2} / \mathrm{Pt}^{0}, E^{0}=1.20 \mathrm{~V}\right)$ to spontaneously reduce $\mathrm{Au}^{+3}$ species under standard conditions. To investigate if the Pt NCs indeed suffer an etching and dissolution during the formation of heterodimers due to their reductive role, the experiment of figure 1 was repeated both in the presence and absence of $\mathrm{Au}^{+3}$ precursor for $24 \mathrm{~h}$. The samples were destabilized by the addition of EtOH (3-5 mL) and centrifuged to precipitate any suspended particle. Chemical analysis of the resulting supernatant by ICP-MS did not show a significant presence of dissolved $\mathrm{Pt}^{+2}$ in neither of the solutions, with only $0.2 \%$ and $0.3 \%$ of the mass found from the initial Pt NCs in the absence and presence of $\mathrm{Au}^{+3}$, respectively. These results further corroborate the role of OAM as the reducer in these reactions. However, for high initial concentrations of the reagents Pt NCs and $\mathrm{Au}^{+3}$, the corrected potential given by the Nerst equation $\left[\mathrm{E}=\mathrm{E}^{\circ}-(\mathrm{RT} / n \mathrm{~F}) \ln \mathrm{Q}\right]$ could make the reaction to happen spontaneously, which would explain the initial decrease of the Pt NCs size upon the heterodimer formation (Figure S3, ESI).

Figure 2 shows the heterodimer structures synthesized in the presence of different $\mathrm{Au}^{+3}$ precursor concentrations while maintaining a constant Pt NC concentration of [10 nM] (for quantities see Table S1, ESI). The heterodimers became progressively more apparent as the initial $\mathrm{Au}^{+3}$ concentration increased, and the mean diameter of the corresponding Au domains increased from $7.9 \pm 1.0 \mathrm{~nm}$ (Figure 2A) to 9.1 $\pm 1.4 \mathrm{~nm}$ (Figure 2E). These results show that the growth of Au NCs is directly related to the increased availability of the $\mathrm{Au}^{+3}$ ions to be reduced on top of the Au domains. By the contrary, when the Pt seed 
concentration was decreased by 10x (Figure 2F) while maintaining a Au precursor concentration similar to that in figure 2C, the size of the Au NCs obtained was $10.6 \pm 1.9 \mathrm{~nm}$, along with the observation of some free Au NCs. Please note, that under any set of conditions we manage to fully dissolve the initial Pt NCs, which supports the role of OAM as the main reducer in these reactions. However, in all the cases the nucleation of Au was drastically accelerated by the presence of Pt NCs.

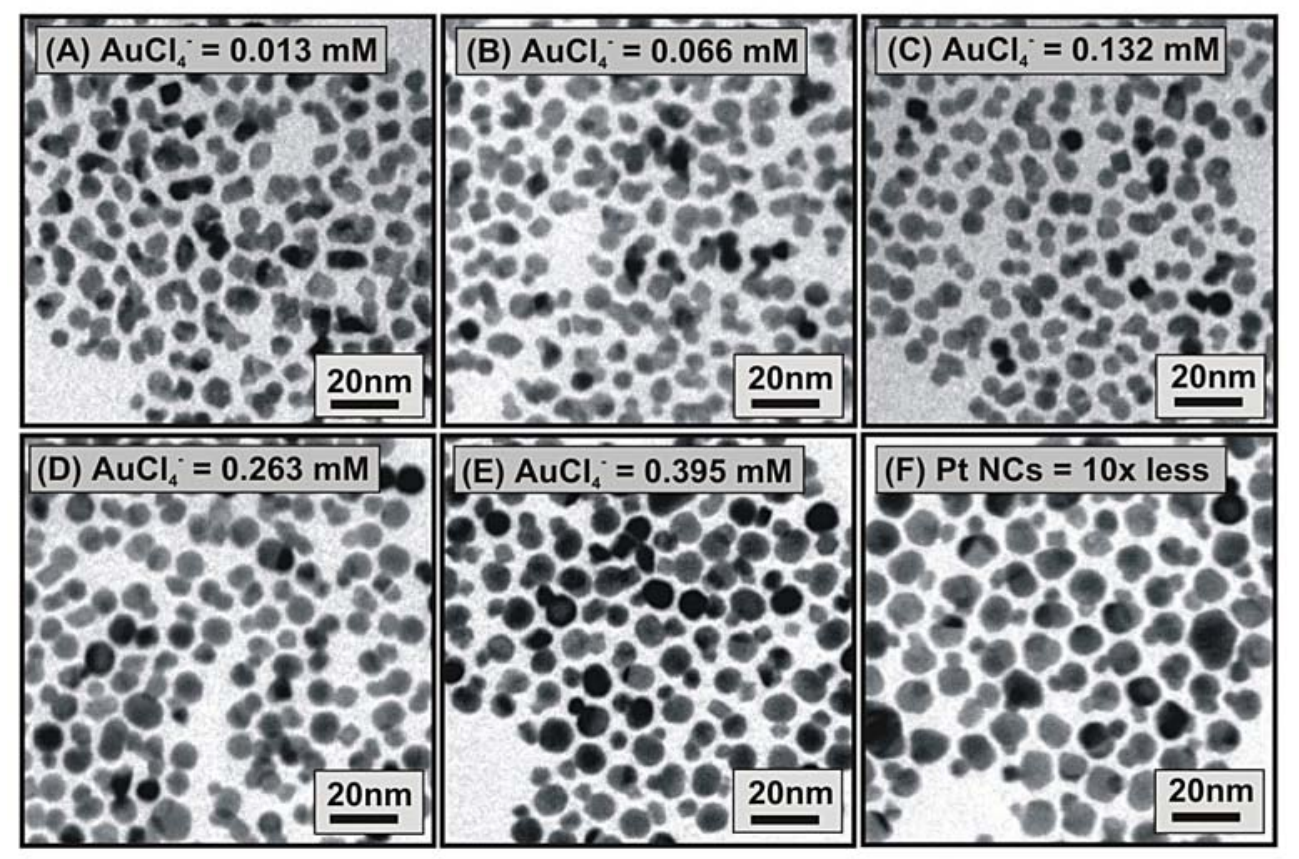

Fig. 2. TEM images of the heterodimers with increasing $\mathrm{Au}^{+3}$ precursor concentration (A-E) or decrease of Pt NCs (F), sampled after $96 \mathrm{~h}$ of reaction.

Structural Characterization. From figure 3A, it is evident that most of the NCs are present in the heterodimer form. Additional HRTEM analysis showed how the Au-domains grow epitaxially on top of the Pt seeds (Figure 3B). Some of the Au NCs in the heterodimers exhibit twin dislocations and are faceted (the five triangular top facets of the Au decahedron are marked with dashed lines). Fast-Fourier transform (FFT) power spectrum analysis shows that both NCs are oriented along the [110] zone axis, with the inner and outer dashed circle marking the $\{111\}$ and $\{002\}$ Au planes, respectively. Notice that 
the FFT pattern also presents the typical striking lines for such twinned and faceted NCs, and that the cell parameters observed in the HRTEM micrographs coincide with those calculated for the fcc structure of Au. In order to confirm the changes in the cell parameter or fringe deformation due to the chemical variation along the NCs, Geometrical Phase Analysis (GPA) ${ }^{21}$ of the heterodimers was carried out (Figure 3C). Taking into account that the interplanar distances of the $\{111\}$ planes in Au and Pt are 0.236 and $0.226 \mathrm{~nm}$ respectively, we would expect a variation of $\sim 4 \%$ in the fringe distance between both parts of the heterodimer system. Results obtained with GPA clearly match with the predicted variations and show the two different metals with the fringe deformation in color maps (\%). Due to the close proximity of Au and Pt in the periodic table and the small size of the NCs, the characteristic X-ray emissions were difficult to resolve using energy dispersive X-ray spectroscopy. In the case of electron energy loss spectroscopy, the high energy of the relative edges also makes difficult to discern between both signals.

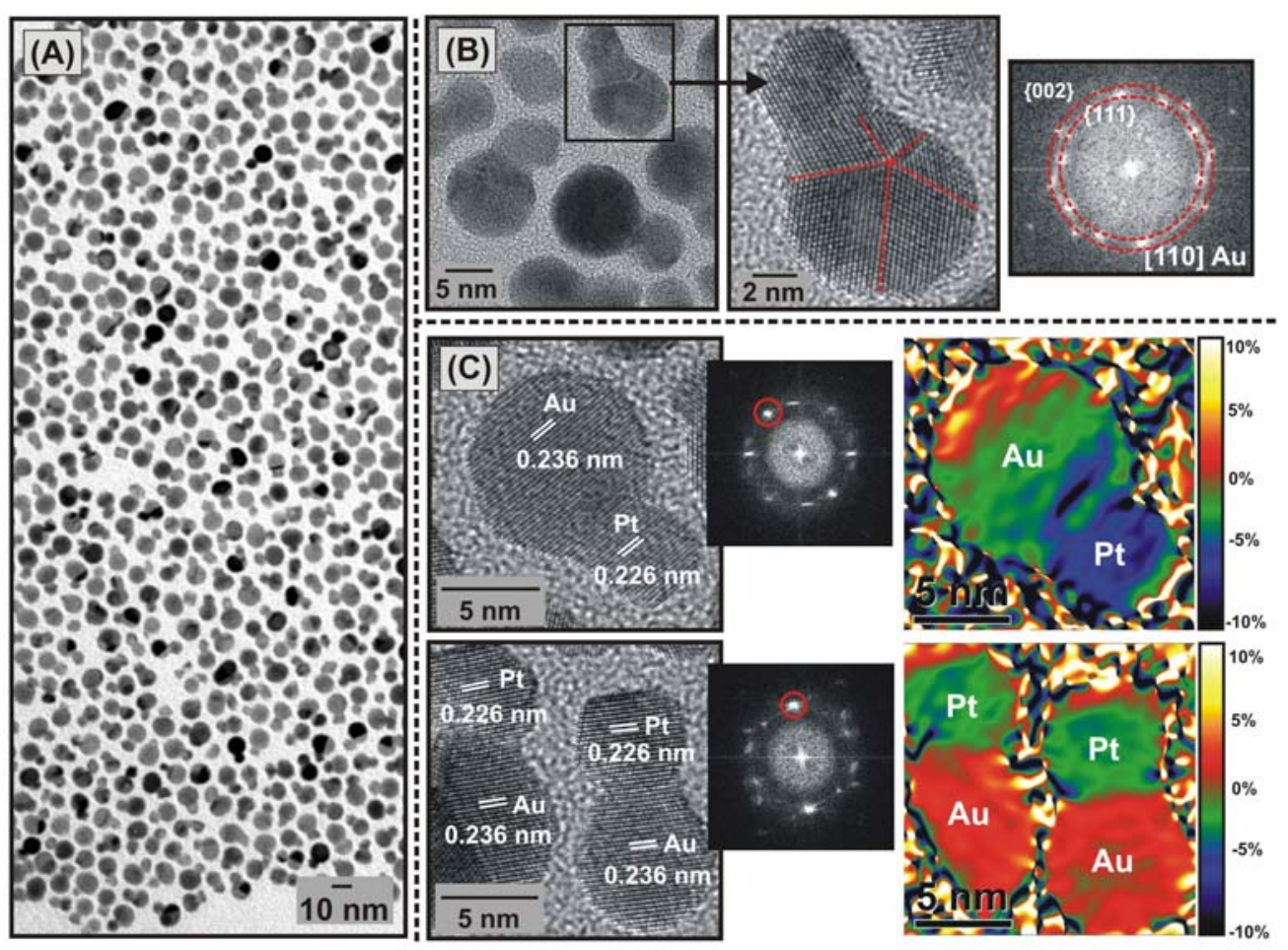

Fig. 3. (A) Low and (B) high resolution TEM images; and (C) GPA of the heterodimers with the corresponding FFT power spectra. The red circles in the FFT indicate the (111) spot used for the GPA. 
The crystalline structures of the heterodimers were also characterized by powder XRD and compared to the diffraction pattern of individual Au and Pt NCs (Figure 4). The experimentally determined XRD spectrum of the heterodimer structures show a mixture of the two patterns originating from nanosized Au and Pt particles, as for example with the peak corresponding to the (111) planes at $2 \theta=38.22^{\circ}(\mathrm{Au})$ and $40.04^{\circ}(\mathrm{Pt})$. After comparing in more detail the $2 \theta$ values between the theoretical and the experimentally obtained Au peaks, it could be observed a slight shift towards higher angles in the case of the heterodimer, which suggests that the crystal strain is maintained and does not relax due to the smallness of the crystal domains (Table S2, ESI). In addition, the sharp intensity of the Au (220) peak further confirms the (110) texture of the heterodimers.

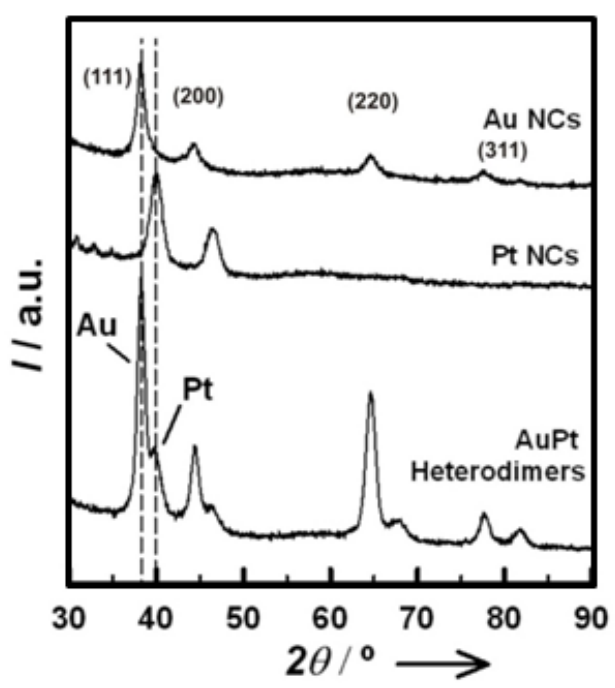

Fig. 4. Powder XRD spectra comparing Au NCs, Pt NCs and heterodimer crystalline structures after 114 $\mathrm{h}$ of reaction at room temperature.

The surface plasmon resonance (SPR) is a direct measurement of the electron cloud of the conforming metals. As shown in figure 5, while Pt NCs do not have a maximum SPR band in the visible range at $0 \mathrm{~h}$ (a), a small band begins to emerge after $10 \mathrm{~h}$ of reaction (c). A maximum SPR peak was observed at 547 
nm after 24 h (d), corresponding to the growth of the attached Au NCs on the Pt seeds. The observed broadening of the SPR band may be due to the lack of monodispersity of the Au NCs, and a combined influence from the electronic effect of the Pt seeds. As time progressed (from e-h), more prominent and narrower SPR bands were observed around 537-543 nm due to the continuous growing of the Au NCs and an increase in the monodispersity of the Au NC size. When examined more closely, we observed a slight blue-shift of $\sim 6 \mathrm{~nm}$ from (e) to (h). This is consistent with the heterodimer evolution since the growth of larger Au NCs red-shifts the plasmon while the presence of Pt induces a charge transfer to the Au and causes blue-shift. A balance between these two trends leads to the observed SPR peak evolution, as depicted in the inset of figure 5. Thus, when more $\mathrm{Au}^{+3}$ precursor was added (i), a red-shift of the band to $544 \mathrm{~nm}$ and an increase in the absorbance was observed. Compared to single component metals, the heterodimers exhibit unique optical properties due to the coupling and electronic effect of the two dissimilar metal domains. While individual Au NCs (5-20 nm in size) exhibit a SPR band around 520 $\mathrm{nm},{ }^{22}$ the heterodimers were detected in the 540-550 nm region. This red-shift suggests an effective coupling, where the Au electronic structure is modified by the presence of Pt seeds, and tunes the overall optical properties. Similar optical properties of these types of heterodimers have also been reported by other researchers. ${ }^{6,9}$ 


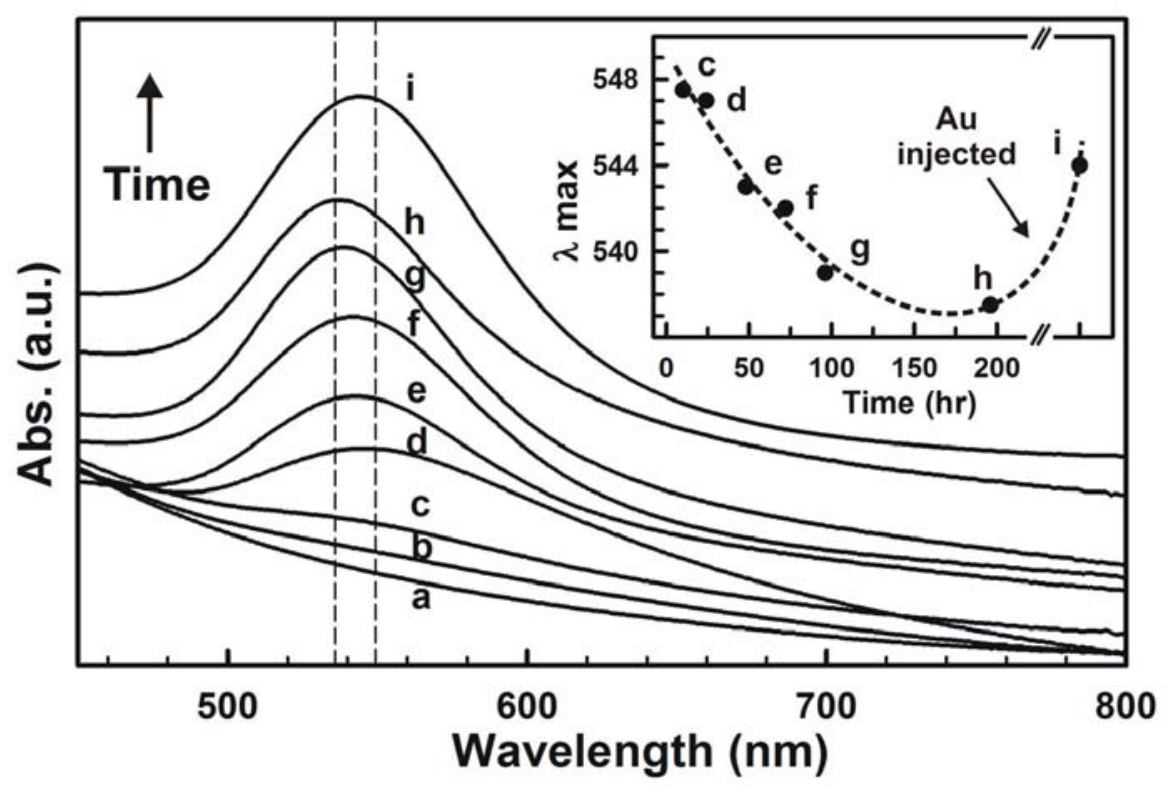

Fig. 5. UV-Vis spectra for the heterodimers sampled at: $0 \mathrm{~h}$ (a), $6 \mathrm{~h}$ (b), $10 \mathrm{~h}$ (c), $24 \mathrm{~h}$ (d), $48 \mathrm{~h} \mathrm{(e),} 72 \mathrm{~h}$ (f), $96 \mathrm{~h} \mathrm{(g),} 196 \mathrm{~h}$ (h) and after additional $\mathrm{Au}^{+3}$ injection (i). (Inset) A plot of maximum absorbance peak ( $\lambda$ ) vs time. The dotted line illustrates the change in $\lambda$.

\section{Conclusions}

We have reported a straightforward method for the synthesis of AuPt heterodimers performed at room temperature using oleylamine as the reducer. The presence of Pt NCs markedly accelerates the nucleation rate of $\mathrm{Au}$, which suggest its role as both nucleation platforms and initial catalytic reducers, as supported by the initial shrinking of the Pt seeds. Room temperature heterodimer synthesis has also been reported by Yang et al., ${ }^{12}$ which illustrated the anisotropic growth of Au nanorods in the presence of Pt nanocubes using ascorbic acid as the reducer. However, a number of crystal defects could be observed at the interface. In our case, the lower reaction temperature induced a slower formation of heterodimer structures, where the sizes and the SPR band shift could more easily be monitored with time. HRTEM images showed an epitaxial growth of Au on Pt. 
Acknowledgement. This work was supported by the Spanish MICINN. S.L. thanks the Catalan Gov. for a B.P. grant, and M.V. for the support from project: MAT2006-13572-C02-02. The authors thank Eudald Casals for help in characterization and the Serveis Cientificotecnics of Universitat de Barcelona (TEM).

Supporting Information Available: Discussion and additional characterization of the heterodimers. This material is available free of charge via the Internet at http://pubs.acs.org. 


\section{References}

(1) Love, J. C.; Estroff, L. A.; Kriebel, J. K.; Nuzzo, R. G.; Whitesides, G. M. Chem. Rev. 2005, 105, 1103.

(2) Daniel, M.-C.; Astruc, D. Chem. Rev. 2004, 104, 293.

(3) Cao, Y.; Banin, U. J. Am. Chem. Soc. 2000, 122, 9692.

(4) Milliron, D. J.; Hughes, S. M.; Cui, Y.; Manna, L.; Li, J.; Wang, L.-W.; Alivisatos, A. P. Nature 2004, 430, 190.

(5) Wang, C.; Daimon, H.; Sun, S. Nano Lett. 2009, 9, 1493.

(6) (a) Wang, C.; Xu, C.; Zeng, H.; Sun, S. Adv. Mater. 2009, 21, 3045. (b) Yu, H.; Chen, M.; Rice, P. M.; Wang, S. X.; White, R. L.; Sun, S. Nano Lett. 2005, 5, 379. (c) Xu, C.; Xie, J.; Ho, D.; Wang, C.; Kohler, N.; Walsh, E. G.; Morgan, J. R.; Chin, Y. E.; Sun, S. Angew. Chem. Int. Ed. 2008, 47, 173. (d) Wang, C.; Tian, W.; Ding, Y.; Ma, Y.-q.; Wang, Z. L.; Markovic, N. M.; Stamenkovic, V. R.; Daimon, H.; Sun, S. J. Am. Chem. Soc. 2010, 132, 6524.

(7) (a) Gu, H.; Yang, Z.; Gao, J.; Chang, C. K.; Xu, B. J. Am. Chem. Soc. 2005, 127, 34.(b) Gu, H.; Zheng, R.; Zhang, X.; Xu, B. J. Am. Chem. Soc. 2004, 126, 5664.

(8) (a) Cozzoli, P. D.; Pellegrino, T.; Manna, L. Chem. Soc. Rev. 2006, 35, 1195. (b) Pellegrino, T.; Fiore, A.; Carlino, E.; Giannini, C.; Cozzoli, P. D.; Ciccarella, G.; Respaud, M.; Palmirotta, L.; Cingolani, R.; Manna, L. J. Am. Chem. Soc. 2006, 128, 6690.

(9) Shi, W.; Zeng, H.; Sahoo, Y.; Ohulchanskyy, T. Y.; Ding, Y.; Wang, Z. L.; Swihart, M.; Prasad, P. N. Nano Lett. 2006, 6, 875.

(10) Teranishi, T.; Saruyama, M.; Nakaya, M.; Kanehara, M. Angew. Chem. Int. Ed. 2007, 46, 1713.

(11) Camargo, P. H. C.; Xiong, Y.; Ji, L.; Zuo, J. M.; Xia, Y. J. Am. Chem. Soc. 2007, 129, 15452.

(12) Habas, S. E.; Lee, H.; Radmilovic, V.; Somorjai, G. A.; Yang, P. Nat. Mater. 2007, 6, 692.

(13) Choi, J.-s.; Choi, H. J.; Jung, D. C.; Lee, J.-H.; Cheon, J. Chem. Commun. 2008, 2197.

(14) Kwon, K.-W.; Shim, M. J. Am. Chem. Soc. 2005, 127, 10269.

(15) Yang, J.; Elim, H. I.; Zhang, Q.; Lee, J. Y.; Ji, W. J. Am. Chem. Soc. 2006, 128, 11921. 
(16) Wetz, F.; Soulantica, K.; Falqui, A.; Respaud, M.; Snoeck, E.; Chaudret, B. Angew. Chem. Int. Ed. 2007, 46, 7079.

(17) Mokari, T.; Rothenberg, E.; Popov, I.; Costi, R.; Banin, U. Science 2004, 304, 1787.

(18) Lim, S. I.; Ojea-Jimenez, I.; Varon, M.; Casals, E.; Arbiol, J.; Puntes, V. Nano Lett. 2010, 10, 964.

(19) Danscher, G. Histochemistry 1981, 71, 81.

(20) Kristian, N.; Wang, X. Electrochem. Commun. 2008, 10, 12.

(21) (a) Hÿtch, M. J.; Snoeck, E.; Kilaas, R. Ultramicroscopy 1998, 74, 131. (b) Johnson, C. L.; Snoeck, E.; Ezcurdia, M.; Rodríguez-González, B.; Pastoriza-Santos, I.; Liz-Marzán, L. M.; Hÿtch, M. J. Nat. Mater. 2008, 7, 120.

(22) Njoki, P. N.; Lim, I.-I. S.; Mott, D.; Park, H.-Y.; Khan, B.; Mishra, S.; Sujakumar, R.; Luo, J.; Zhong, C. J. J. Phys. Chem. C 2007, 111, 14664. 